\title{
Seven-Coordinate Iron and Manganese Complexes with Acyclic and Rigid Pentadentate Chelates and their Superoxide Dismutase Activity
}

Gao-Feng Liu, Miloš Filipović, Frank W. Heinemann and Ivana Ivanović-Burmazović*

Table S1. Analysis of Potential Hydrogen Bonds

$\mathrm{Fe}^{\mathrm{II}} \mathrm{H}_{2}$ Dapsox

\begin{tabular}{|c|c|c|c|c|}
\hline Donor $-\mathrm{H} \cdots$ Acceptor & $d(D-H)$ & $\mathbf{d}(\mathbf{H} \cdots \mathbf{A})$ & $d(D \cdots A)$ & $<(\mathrm{D}-\mathrm{H} \cdots \mathbf{A})$ \\
\hline \multicolumn{5}{|l|}{ Intramolecular } \\
\hline $\mathrm{N}(4)-\mathrm{H}(4 \mathrm{~A}) \cdots \mathrm{O}(1)$ & 0.87 & 2.30 & 2.6660 & 105 \\
\hline $\mathrm{N}(7)-\mathrm{H}(7 \mathrm{E}) \ldots \mathrm{O}(3)$ & 0.83 & 2.39 & 2.7143 & 104 \\
\hline $\mathrm{N}(6)-\mathrm{H}(6 \mathrm{~N}) \ldots \mathrm{O}(4)$ & 0.82 & 2.41 & 2.6923 & 101 \\
\hline $\mathrm{O}(6)-\mathrm{H}(6 \mathrm{~B}) \ldots \mathrm{O}(12)$ & 0.82 & 1.91 & 2.7258 & 170 \\
\hline $\mathrm{N}(4)-\mathrm{H}(4 \mathrm{~A}) \cdots \mathrm{O}(11)$ & 0.87 & 2.64 & 3.4862 & 163 \\
\hline $\mathrm{N}(7)-\mathrm{H}(7 \mathrm{E}) \ldots \mathrm{O}(11)$ & 0.83 & 2.19 & 3.0016 & 164 \\
\hline \multicolumn{5}{|l|}{ Intermolecular } \\
\hline $\mathrm{N}(3)-\mathrm{H}(3 \mathrm{~N}) \cdots \mathrm{O}(7)(\mathrm{a})$ & 0.86 & 1.97 & 2.8182 & 170 \\
\hline $\mathrm{N}(4)-\mathrm{H}(4 \mathrm{~A}) \cdots \mathrm{O}(13)(\mathrm{b})$ & 0.87 & 2.56 & 3.0265 & 115 \\
\hline $\mathrm{N}(4)-\mathrm{H}(4 \mathrm{~B}) \cdots \mathrm{O}(13)(\mathrm{c})$ & 0.85 & 2.09 & 2.9088 & 164 \\
\hline $\mathrm{N}(4)-\mathrm{H}(4 \mathrm{~B}) \cdots \mathrm{O}(4)(\mathrm{d})$ & 0.85 & 2.58 & 3.0322 & 115 \\
\hline $\mathrm{O}(5)-\mathrm{H}(5 \mathrm{~A}) \cdots \mathrm{O}(22)(\mathrm{e})$ & 0.84 & 1.91 & 2.7304 & 164 \\
\hline $\mathrm{O}(5)-\mathrm{H}(5 \mathrm{~B}) \cdots \mathrm{O}(21)(\mathrm{d})$ & 0.83 & 1.93 & 2.7273 & 161 \\
\hline
\end{tabular}




\begin{tabular}{|l|l|l|l|l|}
\hline $\mathrm{O}(6)-\mathrm{H}(6 \mathrm{~A}) \ldots \mathrm{O}(23)(\mathrm{b})$ & 0.82 & 1.98 & 2.7810 & 166 \\
\hline $\mathrm{N}(6)-\mathrm{H}(6 \mathrm{~N}) \ldots \mathrm{O}(12)(\mathrm{f})$ & 0.82 & 2.37 & 3.0508 & 141 \\
\hline $\mathrm{N}(6)-\mathrm{H}(6 \mathrm{~N}) \ldots \mathrm{O}(2)(\mathrm{g})$ & 0.82 & 2.45 & 2.9778 & 123 \\
\hline $\mathrm{N}(7)-\mathrm{H}(7 \mathrm{D}) \ldots \mathrm{O}(6)(\mathrm{b})$ & 0.83 & 2.45 & 3.0074 & 126 \\
\hline $\mathrm{N}(7)-\mathrm{H}(7 \mathrm{D}) \ldots \mathrm{O}(7)(\mathrm{b})$ & 0.83 & 2.29 & 2.9683 & 139 \\
\hline $\mathrm{O}(7)-\mathrm{H}(7 \mathrm{~F}) \ldots \mathrm{O}(2)(\mathrm{f})$ & 0.83 & 1.93 & 2.7020 & 155 \\
\hline $\mathrm{O}(7)-\mathrm{H}(7 \mathrm{G}) \ldots \mathrm{O}(4)(\mathrm{h})$ & 0.80 & 2.02 & 2.7571 & 152 \\
\hline
\end{tabular}

(a) $\mathrm{x}, 1 / 2-\mathrm{y},-1 / 2+\mathrm{z}$; (b) $-1+\mathrm{x}, \mathrm{y}, \mathrm{z}$; (c) $-\mathrm{x}, 1-\mathrm{y},-\mathrm{z}$; (d) $-1+\mathrm{x}, 1 / 2-\mathrm{y},-1 / 2+\mathrm{z}$; (e) $1-\mathrm{x},-1 / 2+\mathrm{y}, 1 / 2-\mathrm{z}$; (g) $\mathrm{x}, 1 / 2-\mathrm{y}, 1 / 2+\mathrm{z}$; (g) $-\mathrm{x},-1 / 2+\mathrm{y}, 1 / 2-\mathrm{z}$; (h) $2-\mathrm{x},-1 / 2+\mathrm{y}, 1 / 2-\mathrm{z}$

$\mathrm{Mn}^{\mathrm{II}} \mathrm{H}_{2}$ Dapsox

\begin{tabular}{|c|c|c|c|c|}
\hline Donor $-\mathrm{H} \cdots$ Acceptor & d(D-H) & $\mathbf{d}(\mathbf{H} \cdots \mathbf{A})$ & $d(D \cdots A)$ & $<(\mathrm{D}-\mathrm{H} \cdots \mathbf{A})$ \\
\hline \multicolumn{5}{|l|}{ Intramolecular } \\
\hline $\mathrm{N}(4)-\mathrm{H}(4 \mathrm{C}) \ldots \mathrm{O}(1)$ & 0.89 & 2.39 & 2.7031 & 101 \\
\hline $\mathrm{N}(7)-\mathrm{H}(7 \mathrm{D}) \ldots \mathrm{O}(3)$ & 0.90 & 2.47 & 2.7629 & 100 \\
\hline $\mathrm{N}(6)-\mathrm{H}(6 \mathrm{C}) \ldots \mathrm{O}(4)$ & 0.89 & 2.22 & 2.6303 & 108 \\
\hline $\mathrm{O}(6)-\mathrm{H}(6 \mathrm{~A}) \ldots \mathrm{O}(3)$ & 0.84 & 2.16 & 3.03 & 163 \\
\hline $\mathrm{N}(4)-\mathrm{H}(4 \mathrm{C}) \ldots \mathrm{O}(23)$ & 0.89 & 2.10 & 2.9237 & 154 \\
\hline $\mathrm{N}(7)-\mathrm{H}(7 \mathrm{D}) \ldots \mathrm{O}(24)$ & 0.90 & 2.26 & 3.1360 & 164 \\
\hline $\mathrm{N}(7)-\mathrm{H}(7 \mathrm{D}) \ldots \mathrm{O}(22)$ & 0.90 & 2.52 & 3.5776 & 155 \\
\hline \multicolumn{5}{|l|}{ Intermolecular } \\
\hline $\mathrm{N}(3)-\mathrm{H}(3 \mathrm{~B}) \ldots \mathrm{O}(4)(\mathrm{a})$ & 0.89 & 2.04 & 2.9170 & 170 \\
\hline $\mathrm{N}(4)-\mathrm{H}(4 \mathrm{~B}) \ldots \mathrm{O}(21)(\mathrm{e})$ & 0.89 & 2.16 & 3.0404 & 170 \\
\hline $\mathrm{O}(5)-\mathrm{H}(5 \mathrm{~A}) \ldots \mathrm{O}(24)(\mathrm{c})$ & 0.87 & 1.92 & 2.7732 & 171 \\
\hline $\mathrm{O}(6)-\mathrm{H}(6 \mathrm{~A}) \ldots \mathrm{O}(14)(\mathrm{d})$ & 0.84 & 2.02 & 2.7902 & 152 \\
\hline $\mathrm{O}(6)-\mathrm{H}(6 \mathrm{~B}) \ldots \mathrm{O}(11)(\mathrm{a})$ & 0.84 & 1.92 & 2.7014 & 154 \\
\hline $\mathrm{N}(7)-\mathrm{H}(7 \mathrm{E}) \ldots \mathrm{O}(2)(\mathrm{b})$ & 0.89 & 2.03 & 2.8465 & 151 \\
\hline $\mathrm{C}(2)-\mathrm{H}(2 \mathrm{~A}) \ldots \mathrm{O}(13)(\mathrm{c})$ & 0.91 & 2.39 & 3.2145 & 151 \\
\hline $\mathrm{C}(7)-\mathrm{H}(7 \mathrm{~A}) \ldots \mathrm{O}(4)(\mathrm{a})$ & 0.96 & 2.44 & 3.1111 & 126 \\
\hline $\mathrm{C}(11)-\mathrm{H}(11 \mathrm{C}) \ldots \mathrm{O}(13)(\mathrm{d})$ & 0.95 & 2.50 & 3.0487 & 117 \\
\hline
\end{tabular}

(a) $\mathrm{x},-1+\mathrm{y}, \mathrm{z}$; (b) $\mathrm{x}, 1+\mathrm{y}, \mathrm{z}$; (c) $\mathrm{x}, 1 / 2-\mathrm{y}, 1 / 2+\mathrm{z}$; (d) -x,-1/2+y,1/2-z; (e) 1-x,-y,-z 
Figure S1 Cyclic voltammogramms of $\left[\mathrm{Fe}^{\mathrm{III}}(\right.$ dapsox $\left.)\left(\mathrm{H}_{2} \mathrm{O}\right)_{2}\right] \mathrm{ClO}_{4}$ purged with dioxygen in DMSO. Conditions: $[$ complex $]=0 \mathrm{M}$ , $1 \times 10^{-5} \mathrm{M}$ , $5 \times 10^{-5} \mathrm{M}$ , $7.5 \times 10^{-5}$ $\mathrm{M}, 1 \times 10^{-4} \mathrm{M}$ ; $\left[\mathrm{Bu}_{4} \mathrm{NBF}_{4}\right]=0.1 \mathrm{M}, T=298 \mathrm{~K}$, scan rates $=0.2 \mathrm{~V} / \mathrm{s}$.

Figure S2-a Cyclic voltammogramm of $\left[\mathrm{Mn}^{\mathrm{II}}\left(\mathrm{Me}_{2}[15]\right.\right.$ pyridinaneN $\left.\left.\mathrm{N}_{5}\right)\left(\mathrm{H}_{2} \mathrm{O}\right)_{2}\right] \mathrm{Cl}_{2}$ purged with $\mathrm{N}_{2}$, Conditions: $[$ complex $]=1 \times 10^{-3} \mathrm{M}, \mathrm{pH}=7.8(\mathrm{HEPES}),\left[\mathrm{NaClO}_{4}\right]=0.1 \mathrm{M}, T$ $=298 \mathrm{~K}$, scan rates $=0.2 \mathrm{~V} / \mathrm{s}$.

Figure S2-b Cyclic voltammogramm of $\left[\mathrm{Mn}^{\mathrm{II}}\left(\mathrm{H}_{2}\right.\right.$ dapsox $\left.)\left(\mathrm{H}_{2} \mathrm{O}\right)\left(\mathrm{CH}_{3} \mathrm{OH}\right)\right]\left(\mathrm{ClO}_{4}\right)_{2}$ purged with $\mathrm{N}_{2}$, Conditions: $\left[\right.$ complex] $=1 \times 10^{-3} \mathrm{M}, \mathrm{pH}=7.8(\mathrm{HEPES}),\left[\mathrm{NaClO}_{4}\right]=$ 
$0.1 \mathrm{M}, T=298 \mathrm{~K}$, scan rates $=0.2 \mathrm{~V} / \mathrm{s}$.

Figure S3 Cyclic voltammogramm of $\left[\mathrm{Mn}^{\mathrm{II}}\left(\mathrm{Me}_{2}[15]\right.\right.$ pyridinaneN $\left.\left.\mathrm{N}_{5}\right)\left(\mathrm{H}_{2} \mathrm{O}\right)_{2}\right] \mathrm{Cl}_{2}$ purged with dioxygen in DMSO, Conditions: $[$ complex $]=1 \times 10^{-3} \mathrm{M},\left[\mathrm{Bu}_{4} \mathrm{NClO}_{4}\right]=0.1 \mathrm{M}, T$ $=298 \mathrm{~K}$, scan rates $=0.5 \mathrm{~V} / \mathrm{s}$.

Figure S1 


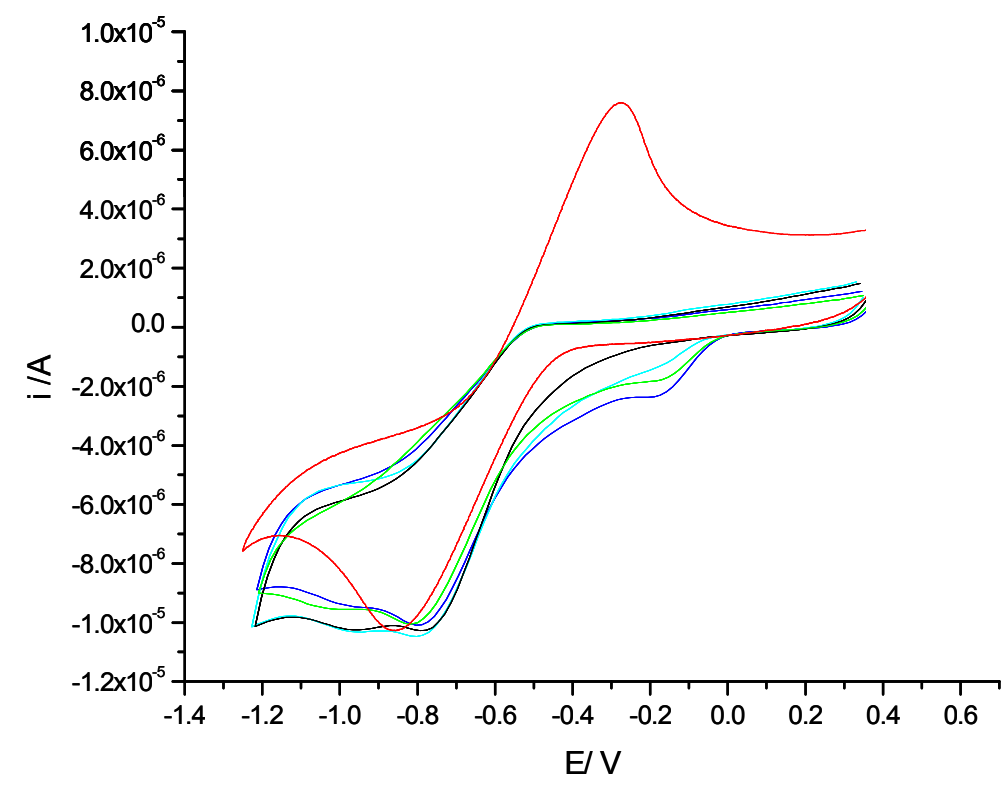

Figure S2-a

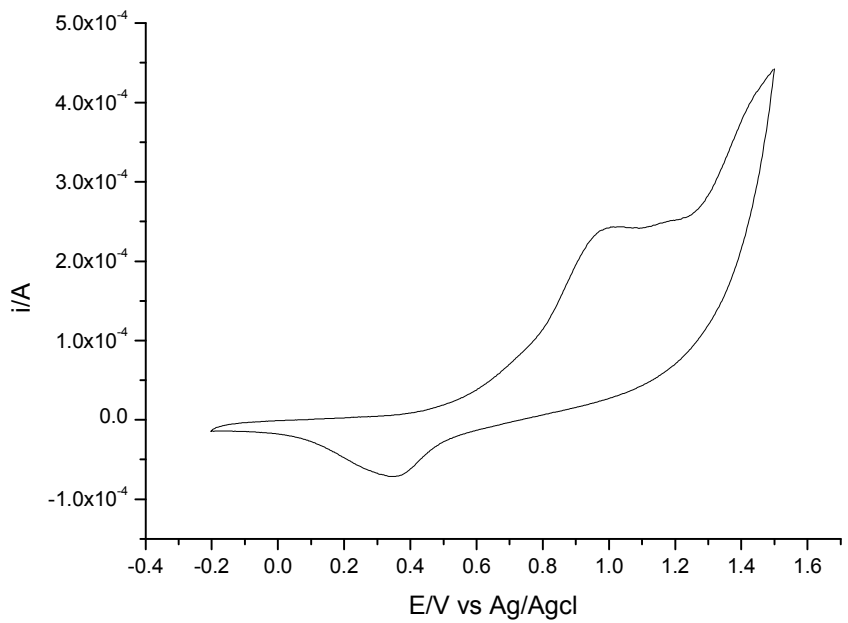

Figure S2-b 


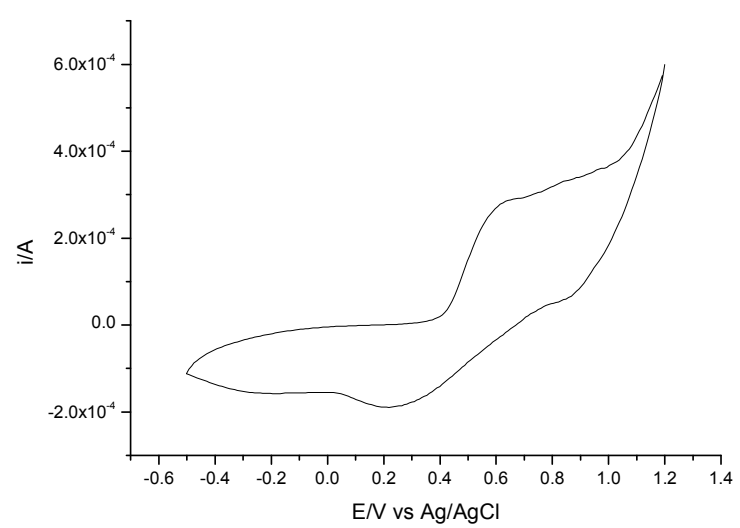

Figure S3

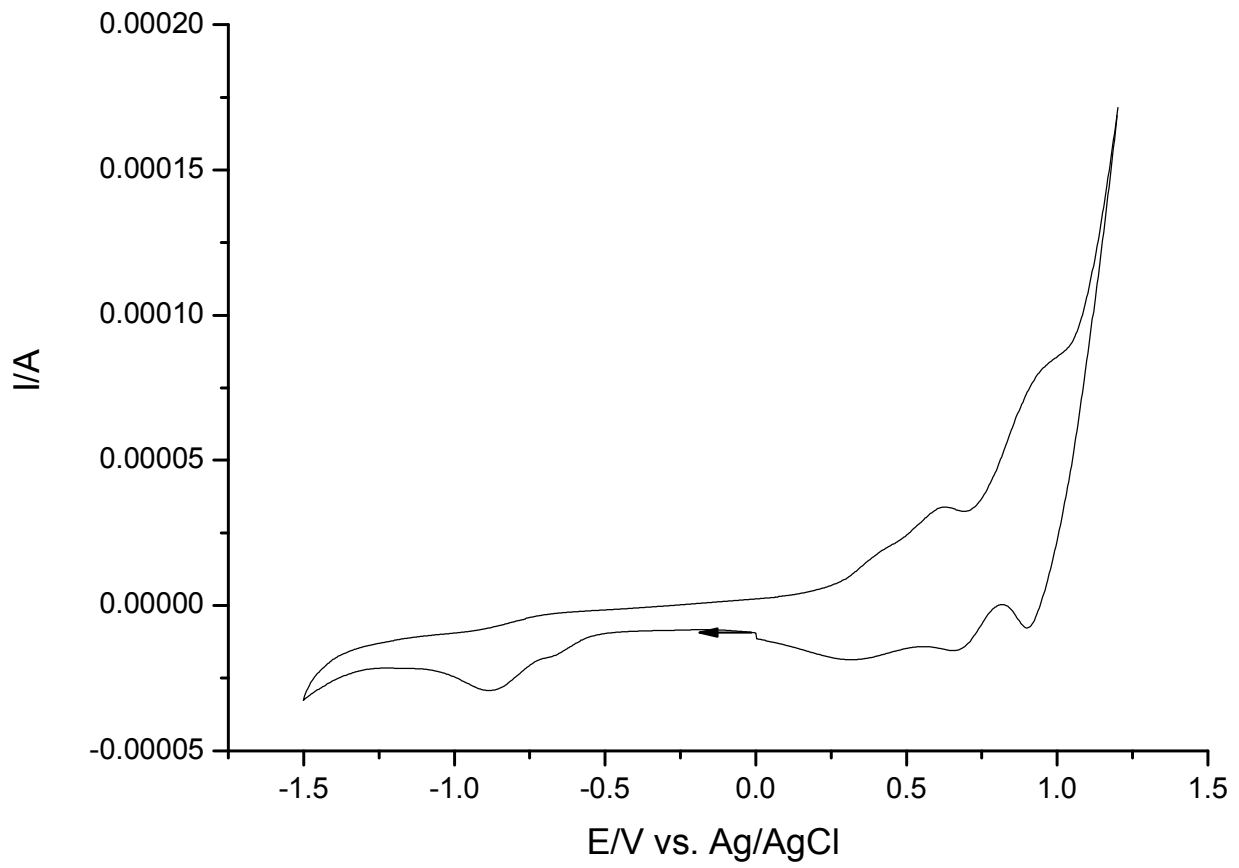

